\title{
MUC1 Expression in Gastric Adenocarcinomas: Its Prognostic Significance and Clinicopathological Correlation
}

\author{
Nisha Raj ${ }^{1}$, Ashok Kumar ${ }^{2}$, Praveer Rai ${ }^{3}$ and Ram Nawal Rao ${ }^{4 *}$ \\ ${ }^{1}$ Department of Pathology, Sanjay Gandhi Post Graduate Institute of Medical \\ Sciences, Lucknow, Uttar Pradesh, India \\ ${ }^{2}$ Department of Surgical Gastroenterology, Sanjay Gandhi Post Graduate Institute \\ of Medical Sciences, Lucknow, Uttar Pradesh, India \\ ${ }^{3}$ Department of Gastroenterology, Sanjay Gandhi Post Graduate Institute of \\ Medical Sciences, Lucknow, Uttar Pradesh, India \\ ${ }^{4}$ Professor, Department of Pathology, Sanjay Gandhi Postgraduate Institute of \\ Medical Sciences, Lucknow, Uttar Pradesh, India \\ *Corresponding Author: Ram Nawal Rao, Professor, Department of Pathology, \\ Sanjay Gandhi Postgraduate Institute of Medical Sciences, Lucknow, Uttar Pradesh, \\ India.
}

\begin{abstract}
Background: Gastric adenocarcinoma, a biologically heterogeneous disease that involves numerous genetic and epigenetic alterations. MUC1 (Mucin-1) acts as an oncoprotein is a membrane associated glycoprotein, has a role in protecting gastric epithelial cells from a variety of external insults which causes inflammation and carcinogenesis. It also functions in cell signaling pathway leading to the upregulation of cyclin D1. In this study, we evaluated the prognostic significance of MUC1 in gastric adenocarcinoma and its association with clinicopathological parameters.

Methods: A total of 70 patients of gastric and gastroesophageal adenocarcinoma were enrolled in to this study. MUC1 protein expression were evaluated by immunohistochemistry using MUC1 antibody on formalin fixed paraffin embedded tissue samples.

Results: Out of 70 cases, there were $43(61.4 \%)$ males and $27(38.6 \%)$ females with a mean age of $54.71 \pm 1.78$ years. Tumors in antropyloric region 33(63.4\%) formed the major bulk. Moderately differentiated adenocarcinoma was predominant in 30(42.8\%) cases followed by poorly differentiated $26(37.2 \%)$ and well differentiated type $14(20 \%)$. MUC1 positivity rate was observed in $47.2 \%$ of cases. Frequency of MUC1 positivity was higher in cases with intestinal type tumors 22(55\%). MUC1 positivity was significantly associated with advanced age, gender, smoking, histological subtypes and with lymph node metastasis ( $p<0.05$ ).We did not find significant correlation of MUC1 expression with tobacco chewer, tumor site, tumor differentiation, Lymphovascular invasion (LVI), perineural invasion(PNI) and TNM staging ( $\mathrm{p}>0.05)$.

Conclusion: MUC1 gene was highly expressed in patients with gastric adenocarcinoma in our study. These findings may of prognostic value and may give some insight in therapeutic decision-making.
\end{abstract}

Keywords: Gastric Adenocarcinomas (GAC); Mucin-1(MUC1); Immunohistochemistry (IHC); Haematoxylin and Eosin (H\&E)

Citation: Ram Nawal Rao., et al. "MUC1 Expression in Gastric Adenocarcinomas: Its Prognostic Significance and Clinicopathological Correlation". Acta Scientific Gastrointestinal Disorders 4.4 (2021): 20-27. 


\section{Introduction}

According to Globocan [1], over one million new cases of gastric cancer were estimated to have occurred in 2018, making it the fifth most common malignancy and third leading cause of cancer mortality ( $8 \%$ of all cancer deaths), mainly because of advanced stage at diagnosis. The most common histological subtype is Gastric adenocarcinoma (GAC). Adenocarcinoma comprises 95\% of the total number of gastric malignancies. Despite the decline in the overall incidence of gastric cancer (GC) in recent decades, it still represents the second leading cause of cancer related death [2].There is a wide geographical variation in both patterns of incidence and survival [3]. Highest mortality rates have been reported in East Asia, including Japan, Korea and China (28.1 per 1000,000 males, 13.0 per 100,000 females) [4]. More than 21,000 new cases of gastric cancer are reported with more than 10,000 deaths annually in United States [5]. In India, the number of new gastric cancer patients is approximately 34000 each year with male to female ratio of 2:1 [6]. In recent years, efforts have been focused on identifying better molecular targets for treatments that interfere with the signaling cascades involved in cell differentiation, proliferation and survival.

Mucins are high molecular weight glycoproteins expressed by specialized epithelial cells lining the luminal surface of different organs like breast, pancreas, respiratory, gastrointestinal and reproductive tracts. Mucins proteins are well known for providing protection and lubrication to epithelial surfaces. In addition, their roles in cell signaling are under intense study $[7,8]$. Variations in mucin expression and glycosylation are associated with cancer development [9]. Atypical expression of mucins is likely associated with cancer biology as alterations in the glycosylation patterns influence cellular growth, differentiation, transformation, adhesion, invasion and immune surveillance [10].

It has been reported that MUC1 increases invasion and metastasis in various cancers. The role of MUC1 in both transformation and metastatic progression has led to extensive focus on this protein for the development of targeted therapies to treat metastatic diseases. Several study reported that MUC1 is a poor prognostic indicator, whereas other reports failed to find its association with different clinicopathological parameters [11-15].

There are still controversies between results of MUC1 expression and their association with different prognostic factors [16-20] and limited number of studies are available worldwide on MUC1 status in gastric cancer patients [21-24]. The objective of the present study was to identify and evaluate the frequency of immunohistochemical expression of MUC1 in gastric adenocarcinoma and to evaluate their correlation with the various clinical and histopathological factors.

\section{Materials and Methods}

Patients and tissue specimens

This was a prospective study of 70 histologically proven patients of gastric or gastro esophageal junction adenocarcinomas who underwent surgery between 2015 to 2018 at Sanjay Gandhi postgraduate institute of medical sciences, Lucknow, India, a tertiary care referral hospital. None of the patients had undergone prior preoperative radiation, chemotherapy or targeted therapy.

\section{Histopathological study}

All specimens were fixed in $10 \%$ buffered formalin, within 30 minutes after resection, and for a fixing time of 8-48 hours. Tissues were processed and embedded in paraffin. Tissue blocks were cut in 3-5 $\mu$ thick sections, fixed for $2 \mathrm{hrs}$ at $60^{\circ} \mathrm{C}$. Deparaffinised sections were stained with haematoxylin and eosin. Histopathological diagnosis were made and adenocarcinomas were classified according to Lauren classification into intestinal, diffuse and mixed type. All the clinical data including demographic information (age, gender, symptoms and sign) were retrieved from patients file and from the hospital informatics system prospectively. In addition, histologic type, degree of differentiation, depth of tumor invasion, presence of lymphovascular, perineural invasion and lymph node metastasis were recorded on a predesigned proforma.

The TNM stage of all studied patients was done according to $8^{\text {th }}$ edition of AJCC. Tumors were graded into well differentiated, moderately differentiated and poorly differentiated as per WHO grading system.

\section{Immunohistochemistry}

Respective paraffin blocks containing representative samples of the tumors were selected by reviewing H\&E stained slides. Paraffin-embedded samples were cut in 3-5 $\mu$ thick sections, taken on poly $\mathrm{L}$ lysine coated slides, fixed for overnight at $60^{\circ} \mathrm{C}$, and then deparaffinized in xylene and hydrated in a decreasing series of alcohol. After those steps, the endogenous peroxidase was blocked 
with 3\% hydrogen peroxidase. Antigen retrieval was performed in a microwave oven for 30 minutes at $98^{\circ} \mathrm{C}$ with the slides immersed in TRIS-EDTA (pH-9). Immunohistochemical staining was performed using Rabbit polyclonal antibody for MUC1 (dilution, 1:100; catalog no., RB9222-P, Thermofisher Scientific, Fremont, CA, USA) followed by addition of diaminobenzidine (DAB) as a chromogen. The slides were stained with hematoxylin for counterstaining and then mounted using DPx. Negative controls (omission of primary antibody) i.e. instead of primary antibody $3 \%$ skimmed milk were used. Positive controls were breast carcinoma tissue and gastric cancer tissue (known IHC MUC1, 3+ staining intensity) were used.

\section{Evaluation of IHC results}

Immunohistochemical evaluation was performed according to testing protocol, taking incomplete or complete cytoplasmic and luminal staining. Slides were scored by a two pathologist using the standard scoring system defined earlier (Hwang I., et al. 2012) table 1. Immunostaining scores were independently evaluated in each tumor core.

Table 1: Scoring method for MUC1 Immunohistochemistry in gastric cancers.

\begin{tabular}{|l|c|c|}
\hline Score & Staining Pattern & $\begin{array}{c}\text { MUC1protein } \\
\text { expression }\end{array}$ \\
\hline 0 & $0 \%$ of tumor cells & Negative \\
\hline $1+$ & $\begin{array}{c}<10 \% \text { of weak Positive } \\
\text { tumor cells }\end{array}$ & Negative \\
\hline $2+$ & $\begin{array}{c}10-50 \% \text { of mild to } \\
\text { moderate Positive tumor } \\
\text { cells }\end{array}$ & Positive \\
\hline $3+$ & $\begin{array}{c}\geq 50 \% \text { of strong Positive } \\
\text { tumor cells }\end{array}$ \\
\hline
\end{tabular}

\section{Statistical analysis}

Statistical analysis was performed by using Statistical Package for the Social Sciences (SPSS Inc., Chicago, IL, USA) 20 package program and results were expressed as mean \pm SD, median (IQR) and in percentage. The MUC1 immunohistochemical expression and its correlation with different parameters was evaluated by using chisquare test and Fischer exact test. P value less than 0.05 were considered statistically significant.

\section{Results}

Demographic and Clinicopathological Parameters

Out of seventy patients enrolled in the study there were $43(61.4 \%)$ female and $27(38.6 \%)$ females, with a mean age of $54.71 \pm 1.78$ years. As per the site of tumor location is concern maximum number of patients had tumor located in antropyloric lesion 33(63.4\%). The second common site of tumor location was at the GE junction $18(25.7 \%)$.

The other site of tumor location were body and fundus $8(15.4 \%)$, body and antrum $11(21.2 \%)$. Patient's clinicopathological parameters are given in (Table 2).

Tumors were graded into well-differentiated tumors included grade I and moderately differentiated tumors grade II adenocarcinomas; poorly differentiated on the other hand, consisted of grade III adenocarcinomas as per WHO grading system.

According to Lauren's classification out of 70 cases $40(57.2 \%)$ were of intestinal type, 22(31.4\%) diffuse type and $8(11.4 \%)$ were of mixed type adenocarcinoma. Majority of tumors had moderately differentiated $30(42.8 \%)$ followed by poorly differentiated $26(37.2 \%)$ and well differentiated tumors $14(20 \%) .0$ f 70 patients, lymph node metastasis (LNM) was reported in $44(62.9 \%)$ patients, while $37(52.8 \%)$ had lymphovascular invasion (LVI). Perineural invasion (PNI) was observed in $30(42.8 \%)$ patients. The TNM stage of all studied patients was done according to $8^{\text {th }}$ edition of AJCC. Stage III and stage II tumors were observed in $24(34.2 \%)$ and $15(21.5 \%)$ cases, respectively.

\section{MUC1 IHC scores of patients}

MUC1 expression analysis showed that majority of patients $33(47.2 \%)$ were of score $3+$ suggesting as MUC1 positivity. A score of $2+$ was seen in 17 (24.2\%) cases i.e. equivocal, while 20(28.6\%) cases showed $0 / 1+$ score, which were considered as negative, shown in figure 1.

MUC1 oncoprotein overexpression was seen predominantly in patients over 60 years of age and higher in male than in female population. MUC1 positivity was found more frequently in moderately differentiated tumors $16 / 30(53.3 \%)$ followed by poorly differentiated $11 / 26(42.3 \%)$ and well differentiated tumors $6 / 14(42.8 \%)$. Higher MUC1 positivity 22/40(55\%) was reported in the cases 


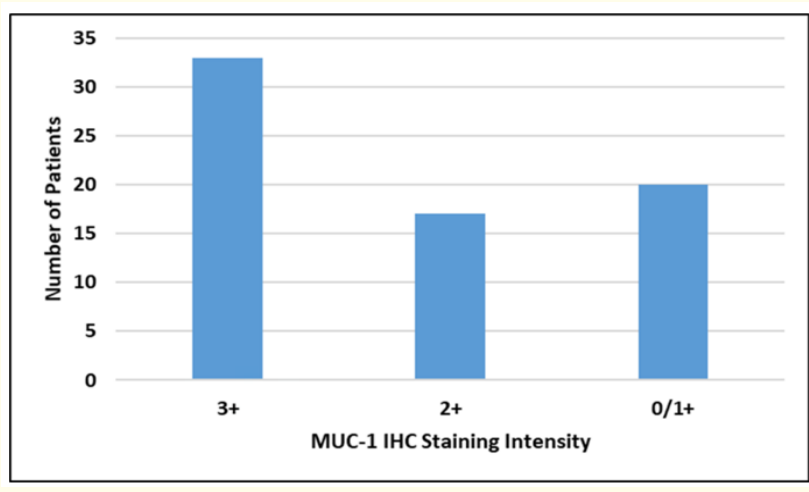

Figure 1: Frequency of MUC1 protein Expression by IHC.

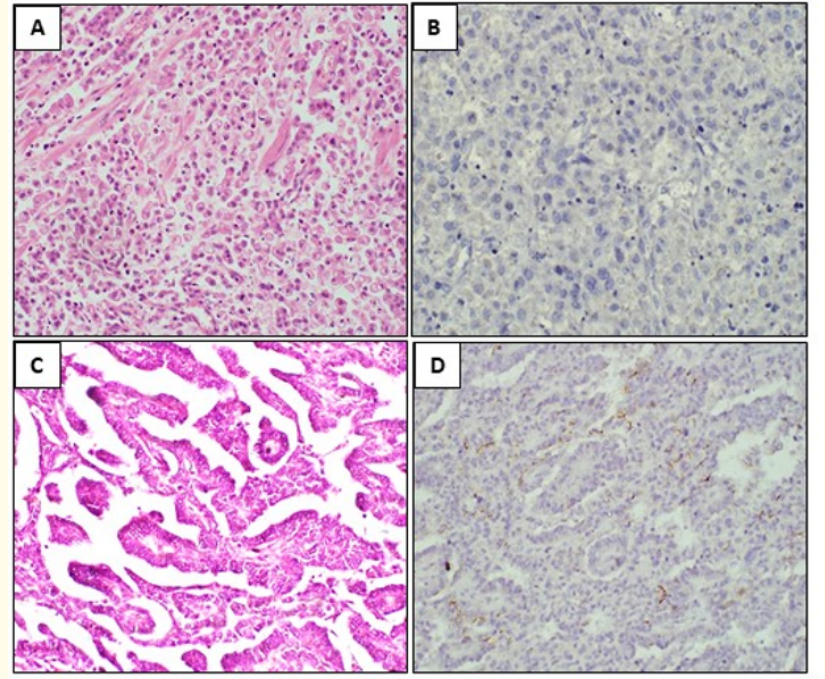

Figure 2: A-B: H\&E stain of Signet ring cell adenocarcinoma and corresponding IHC showing no MUC1 staining in tumor cells (score 0, Negative). C-D: H\&E Stain of Papillary adenocarcinoma and corresponding MUC1 IHC showing $<10 \%$ positive staining in tumor cells (score 1+, Negative).

with intestinal type as compared to diffuse type 8/22(36.3\%) and in mixed type $3 / 8(37.5 \%)$.

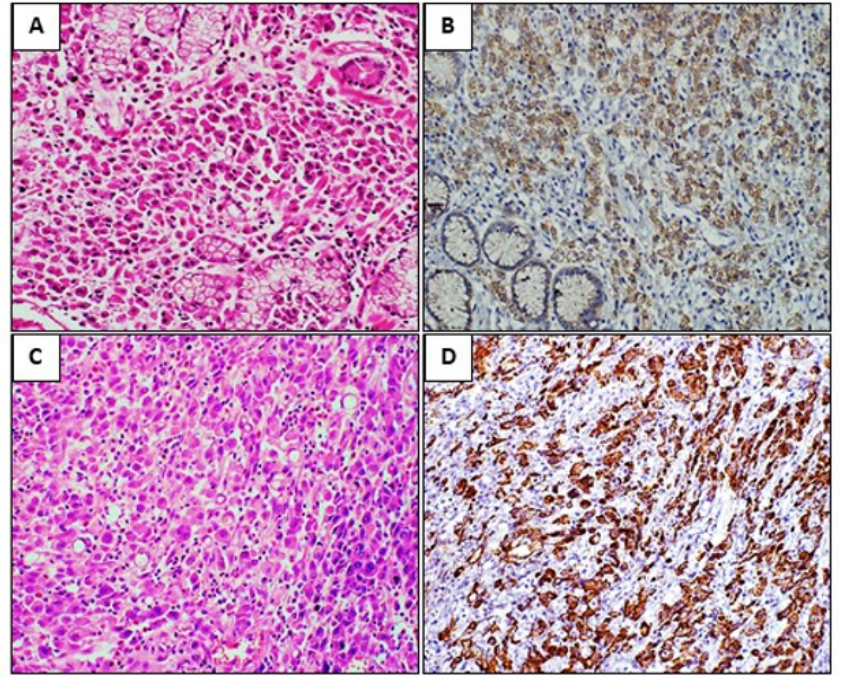

Figure 3: A-B: H\&E stain of Moderately differentiated adenocarcinoma and corresponding IHC showing Moderate cytoplasmic MUC1 immunostaining (score 2+, Equivocal) in tumor cells. C-D: H\&E stain of Signet ring cell adenocarcinoma and corresponding IHC showing Strong membranous and Cytoplasmic MUC1 immunostaining (score 3+ Strong Positive) in tumor cells.

There was significant correlation between MUC1 positivity and poor prognostic histological subtypes $(p=0.010)$. In this study no statistically significant correlation of MUC1 expression was observed with tobacco chewer, tumor site, degree of differentiation, lymphovascular invasion (LVI), perineural invasion (PNI) and with TNM staging ( $\mathrm{p}>0.05)$.

We found that there was significant difference between MUC1 positive and negative patients in terms of age, gender, smoking, histological subtypes and lymph node metastasis (LNM) $(p<0.05)$ as listed in table 2.

\section{Discussion}

Gastric adenocarcinoma is a conglomerate of histologically, biologically and genetically diseases, conditioned by the gradual accumulation of various genetic and epigenetic alterations leading to the activation of several molecular pathways. The prognosis of gastric and gastroesophageal junction cancers is still poor despite re- 
MUC1 Expression in Gastric Adenocarcinomas: Its Prognostic Significance and Clinicopathological Correlation

Table 2: Clinicopathological features and its Correlation with MUC1 Expression.

\begin{tabular}{|c|c|c|c|c|c|c|c|}
\hline \multirow{2}{*}{ S. No. } & \multirow{2}{*}{ Clinical Variables } & \multirow{2}{*}{$N=70(\%)$} & \multicolumn{3}{|c|}{ MUC-1 IHC Score } & \multirow{2}{*}{$\mathbf{X}^{2}$} & \multirow{2}{*}{ p-Value } \\
\hline & & & Positive & Equivocal & Negative & & \\
\hline \multirow[t]{3}{*}{1.} & Gender & & & & & 8.83 & $0.012^{*}$ \\
\hline & Male & $43(61.4)$ & 25 & 11 & 7 & & \\
\hline & Female & $27(38.6)$ & 8 & 6 & 13 & & \\
\hline \multirow[t]{3}{*}{2.} & Age & & & & & 7.72 & $0.021^{*}$ \\
\hline & $>60$ & $39(55.7)$ & 21 & 12 & 6 & & \\
\hline & $\leq 60$ & $31(44.3)$ & 12 & 5 & 14 & & \\
\hline \multirow[t]{3}{*}{3.} & Smoker & & & & & 7.26 & $0.026^{*}$ \\
\hline & Yes & $45(64.2)$ & 24 & 13 & 8 & & \\
\hline & No & $25(35.8)$ & 9 & 4 & 12 & & \\
\hline \multirow[t]{3}{*}{4.} & Tobacco chewer & & & & & 1.17 & 0.557 \\
\hline & Yes & $25(35.7)$ & 10 & 6 & 9 & & \\
\hline & No & $45(64.3)$ & 23 & 11 & 11 & & \\
\hline \multirow[t]{3}{*}{5.} & Tumor site & & & & & 2 & 0.367 \\
\hline & Gastric & $52(74.2)$ & 27 & 12 & 13 & & \\
\hline & GE-Junction & $18(25.8)$ & 6 & 5 & 7 & & \\
\hline \multirow[t]{4}{*}{6.} & Laurens Classification & & & & & 13.1 & $0.010^{*}$ \\
\hline & Intestinal & $40(57.2)$ & 22 & 4 & 14 & & \\
\hline & Diffuse & $22(31.4)$ & 8 & 11 & 3 & & \\
\hline & Mixed type & $8(11.4)$ & 3 & 2 & 3 & & \\
\hline \multirow[t]{4}{*}{7.} & Tumor Differentiation & & & & & 3.43 & 0.486 \\
\hline & Well & $14(20)$ & 8 & 4 & 4 & & \\
\hline & Moderately & $30(42.8)$ & 14 & 10 & 7 & & \\
\hline & Poorly & $26(37.2)$ & 11 & 3 & 9 & & \\
\hline \multirow[t]{3}{*}{8.} & Lymph node metastasis & & & & & 6.29 & $0.043^{*}$ \\
\hline & Yes & $44(62.9)$ & 24 & 12 & 8 & & \\
\hline & No & $26(37.1)$ & 9 & 5 & 12 & & \\
\hline \multirow[t]{3}{*}{9.} & Lymphovascular invasion & & & & & 5.31 & 0.070 \\
\hline & Yes & $37(52.8)$ & 22 & 8 & 7 & & \\
\hline & No & $33(47.2)$ & 11 & 9 & 13 & & \\
\hline \multirow[t]{3}{*}{10.} & Perineural invasion & & & & & 4.46 & 0.107 \\
\hline & Yes & $30(42.8)$ & 18 & 7 & 5 & & \\
\hline & No & $40(57.2)$ & 15 & 10 & 15 & & \\
\hline \multirow[t]{5}{*}{11.} & Staging & & & & & 4.45 & 0.879 \\
\hline & Stage I & $19(27.2)$ & 7 & 5 & 9 & & \\
\hline & Stage II & $15(21.5)$ & 6 & 3 & 4 & & \\
\hline & Stage III & $24(34.2)$ & 14 & 4 & 5 & & \\
\hline & Stage IV & $12(17.1)$ & 6 & 3 & 2 & & \\
\hline
\end{tabular}

Values are presented as number $(\%)$ and mean \pm SD

MUC1 (Mucin1) oncogene, IHC, Immunohistochemistry.

Correlation analysis- *statistically significant with $\mathrm{p}$-value $<0.05$.

Citation: Ram Nawal Rao., et al. "MUC1 Expression in Gastric Adenocarcinomas: Its Prognostic Significance and Clinicopathological Correlation". Acta Scientific Gastrointestinal Disorders 4.4 (2021): 20-27. 
Table 3: Correlation of MUC1 Oncoprotein Expression with Histological subtypes.

\begin{tabular}{|l|c|c|c|c|}
\hline \multirow{2}{*}{$\begin{array}{l}\text { Laurens } \\
\text { Classification }\end{array}$} & \multirow{2}{*}{$\begin{array}{c}\text { No. of } \\
\text { Patients }\end{array}$} & \multicolumn{3}{|c|}{ MUC1 IHC Score } \\
\cline { 3 - 5 } & Positive & Equivocal & Negative \\
\hline Intestinal type & $40(57.2)$ & 22 & 4 & 14 \\
\hline Diffuse type & $22(31.4)$ & 8 & 11 & 3 \\
\hline Mixed type & $8(11.4)$ & 3 & 2 & 3 \\
\hline Total & 70 & 33 & 17 & 20 \\
\hline
\end{tabular}

Cross tabs; Pearson Chi- Square test; $\mathrm{p}$ value $=0.010$ (Significant).

cent advances in treatment. Although the number of patients diagnosed at early stages is increasing, the majority of patients are still being diagnosed in advanced stages or with metastatic diseases.

The role of new molecular targeted agents is being investigated in an effort to improve the survival rate. The investigation of molecular and genetic changes in gastric cancer has brought new insight into the pathogenesis of disease. In cancer tissues, the expression of MUC1 can be upregulated and expressed on the entire cell surface.

The MUC1 positivity rate in our study was observed in $47.2 \%$ of gastric adenocarcinoma cases. However, data reported in literature on MUC1 positivity rates in gastric cancer are variable between 30$60 \%$.Geographical differences, tumor heterogeneity, differences in scoring systems and pathologist expertise may have caused the variations in MUC1 positivity rates between studies. Many studies stated that MUC1 expression was associated with metastatic progression in the gastrointestinal tumors. However, in gastric cancer it was reported that expression of MUC1 was not limited to metastatic disease, but also highly expressed in the majority of isolated cells invading throughout the stroma of primary tumor [19]. Expression pattern of MUC1 in gastric cancer is heterogeneous. This heterogeneity may provide new insights into the differentiation pathways of gastric cancer enabling its use as a clue to bring new insights into biologic behavior of gastric cancer.

Concordance with other reports [25-28], MUC1 showed higher expression rates in intestinal type adenocarcinoma (55\%) in which tumor cell interaction is enhanced, than in diffuse type (36.3\%) in our study. This finding is supported by the fact that a particular carbohydrate epitope of MUC1 binds to a ligand, cell interactions may be enhanced. Similar to [25] study there was significant difference between MUC1 positive and negative patients in terms of histological subtypes $(\mathrm{p}=0.010)$.

In contrast to previous studies, there was significant difference between MUC1 positivity with age, gender, smoking, histological subtypes and with lymph node metastasis [28,29]. However, there was no significant correlation between MUC1 expression with tobacco chewer, tumor site, degree of differentiation, lymphovascular invasion (LVI), perineural invasion (PNI) and TNM staging. This may be due to the non-binding of ligand with epitope of MUC1. On the other hand, previous studies $[10,15,30]$ stated that there were no significant correlations between MUC1 expression and clinicopathological parameters including Laurens classification and degree of differentiation.

MUC1 staining pattern is different depending on tumor differentiation, stain mainly accumulates at the apex in papillary adenocarcinoma, well differentiated or moderately differentiated adenocarcinoma, while poorly differentiated carcinoma and signet ring cell carcinoma demonstrated primarily cytoplasmic staining [31]. In our study, we could not find significant correlation with MUC1 expression and some prognostic factors such as perineural invasion and TNM staging ( $\mathrm{p}>0.05)$.

Our data showed statistically significant correlation between MUC1 positivity and negative patients with age, gender, smoking, histological subtypes and lymph node metastasis $(\mathrm{p}<0.05)$.

\section{Conclusion}

MUC1 gene was highly expressed in patients with gastric adenocarcinomas in our study. Significant correlation was found between MUC1 with several clinical and histological factors. Up-regulated MUC1 expression is closely related with progression and it might be considered as a useful prognostic indicator and may give some insight in therapeutic decision-making. However, further more and larger studies are required to validate these findings.

\section{Conflict of Interest}

The authors declare that they have no any conflict of interest.

\section{Ethics Statement}

The Institutional Ethical Review Committee (IEC number-201721-PhD-95) of Sanjay Gandhi Post Graduate Institute of Medical Sciences in Lucknow, India approved this study. 


\section{Informed Consent}

Informed consent was obtained from all patients included in this study.

\section{Funding}

The authors thank Indian Council of Medical Research, New Delhi, India for providing fellowship support.

\section{Author's Contribution}

Study concept, design, analysis and interpretation of data: Nisha Raj, Dr. Ram Nawal Rao. The authors would like to express their thanks to Dr. Ashok Kumar for help with advanced research and drafting. Dr. Ram Nawal Rao, Department of Pathology, Sanjay Gandhi Post Graduate Institute of Medical Sciences, Lucknow, India for reviewing the histological and immunohistochemical results. All authors read and approved the final manuscript.

\section{Acknowledgements}

The authors acknowledge the patients for sharing the clinical information and samples. We would also like to express our sincere gratitude to Dr. Lily Pal, Dr. Rungmei S.K. Marak and Dr. Kaushik Mandal, from the Departments of Pathology, Microbiology and Medical Genetics, Sanjay Gandhi Post Graduate Institute of Medical Sciences, Lucknow for providing guidance and valuable support in this study.

\section{Bibliography}

1. Bray F., et al. "Global cancer statistics 2018: GLOBOCAN estimates of incidence and mortality worldwide for 36 cancers in 185 countries". CA: A Cancer Journal for Clinicians 68.6 (2018): 394-424.

2. Layke JC and Lopez PP. "Gastric cancer: diagnosis and treatment options". American Family Physician 69.5 (2004): 11331140.

3. Archie V., et al. "Gastric cancer: standards for the 21st century". Critical Reviews in Oncology/Hematology 57.2 (2006): 123-131.

4. Ferlay J., et al. "Estimates of worldwide burden of cancer in 2008: GLOBOCAN 2008". International Journal of Cancer 127.12 (2010): 2893-2917.

5. Siegel R., et al. “Cancer statistics, 2012”. CA: A Cancer Journal for Clinicians 62 (2012): 10-29.
6. Shrikhande SV., et al. "Indian Council of Medical Research consensus document for the management of gastric cancer". Indian Journal of Medical and Paediatric Oncology 35.4 (2014): 239-243.

7. Nakamori S., et al. "MUC1 mucin expression as a marker of progression and metastasis of human colorectal carcinoma". Gastroenterology 106.2 (1994): 353-361.

8. Khodarev NN., et al. "MUC1-induced transcriptional programs associated with tumorigenesis predict outcome in breast and lung cancer". Cancer Research 69.7 (2009): 2833-2837.

9. Song X., et al. "Label-free in vivo molecular imaging of underglycosylated mucin-1 expression in tumour cells". Nature Communication 6 (2015): 6719.

10. Hwang I., et al. "Prognostic significance of membrane-associated mucins 1 and 4 in gastric adenocarcinoma". Experimental and Therapeutic Medicine 4.2 (2012): 311-316.

11. Toki F., et al. "Relationship between clinicopathological features and mucin phenotypes of advanced gastric adenocarcinoma”. World Journal of Gastroenterology 16.22 (2010): 27642770 .

12. Lee OJ., et al. "The prognostic significance of the mucin phenotype of gastric adenocarcinoma and its relationship with histologic classifications". Oncology Report 21.2 (2009): 387-393.

13. Wakatsuki K., et al. "Clinicopathological and prognostic significance of mucin phenotype in gastric cancer". Journal of Surgery on Oncology 98.2 (2008): 124-129.

14. Lee WA., et al. "Genetic expression pattern of gastric carcinomas according to cellular mucin phenotypes". Korean Journal of Pathology 41.5 (2007): 307-315.

15. Kocer B., et al. "Prognostic significance of mucin expression in gastric carcinoma”. Digestive Diseases and Sciences 49.6 (2004): 954-964.

16. Gendler SJ and Spicer AP. "Epithelial mucin genes". Annual Review of Physiology 57 (1995): 607-634.

17. Williams SJ., et al. "Two novel mucin genes down regulated in colorectal cancer identified by differential display". Cancer Research 59.16 (1999): 4083-4089. 
18. Ho SB., et al. "Heterogeneity of mucin gene expression in normal and neoplastic tissues". Cancer Research 53.3 (1993): 641651.

19. Reis CA., et al. "Immunohistochemical study of MUC5AC expression in human gastric carcinomas using a novel monoclonal antibody". International Journal of Cancer 74.1 (1997): 112-121.

20. Carrato C., et al. "Differential apomucin expression in normal and neoplastic human gastrointestinal tissues". Gastroenterology 107.1 (1994): 160-172.

21. Baldus SE., et al. "Correlation of the immunohistochemical reactivity of mucin peptide cores MUC1 and MUC2 with the histopathological subtype and prognosis of gastric carcinomas". International Journal of Cancer 79.2 (1998): 133-138.

22. Baldus SE., et al. "Correlation of MUC5AC immunoreactivity with histopathological subtypes and prognosis of gastric carcinoma”. Annals of Surgical Oncology 9.9 (2002): 887-893.

23. Utsunomiya T., et al. "Expression of MUC1 and MUC2 mucins in gastric carcinomas: its relationship with the prognosis of the patients". Clinical Cancer Research 4.11 (1998): 2605-2614.

24. Gurbuz Y., et al. "How do gastric carcinoma classification systems relate to mucin expression patterns? An immunohistochemical analysis in a series of advanced gastric carcinomas". Virchows Archive 440.5 (2002): 505-511.

25. İlhan 0., et al. "Prognostic significance of MUC1, MUC2 and MUC5AC expressions in gastric carcinoma". Turkish Journal of Gastroenterology 21.4 (2010): 345-352.

26. Akyurek N., et al. "Expression of MUC1 and MUC2 mucins in gastric carcinomas: their relationship with clinicopathologic parameters and prognosis". Pathology - Research and Practice 198.10 (2002): 665-674.

27. Barresi V., et al. "Relationship between immunoexpression of mucin peptide cores MUC1 and MUC2 and Lauren's histologic subtypes of gastric carcinomas". European Journal of Histochemistry: EJH 50.4 (2006): 301-309.

28. Lee HS., et al. "MUC1, MUC2, MUC5AC, and MUC6 expressions in gastric carcinomas: their roles as prognostic indicators". Cancer 92.6 (2001): 1427-1434.
29. Li XH., et al. "The clinicopathological and prognostic significance of MUC-1 expression in Japanese gastric carcinomas: an immunohistochemical study of tissue microarrays". Anticancer Research 28 (2008): 1061-1067.

30. Geramizadeh B., et al. "Mucin profile expression in gastric adenocarcinoma". Middle East Journal of Digestive Diseases 4.4 (2012): 211-215.

31. Yonezawa S., et al. "Expression profiles of MUC1, MUC2, and MUC4 mucins in human neoplasms and their relationship with biological behavior". Proteomics 8.16 (2008): 3329-3341.

\section{Assets from publication with us}

- Prompt Acknowledgement after receiving the article

- Thorough Double blinded peer review

- Rapid Publication

- Issue of Publication Certificate

- High visibility of your Published work

Website: www.actascientific.com/

Submit Article: www.actascientific.com/submission.php Email us: editor@actascientific.com

Contact us: +919182824667 